\title{
Immature Granulocyte Count and Percentage as New Predictors of Mortality in Patients with Upper Gastrointestinal Bleeding
}

\author{
Cihan Bedel ${ }^{1}$, Mustafa Korkut ${ }^{2}$, Ali Avcl $^{3}$, Ahmet Uzun $^{4}$
}

\begin{abstract}
Aims: Early identification of patients at risk of adverse outcomes may increase the survival rates in patients with upper gastrointestinal bleeding (UGIB), but this can be difficult to predict in emergencies. The aim of the study is to evaluate immature granulocyte (IG), which can be obtained from simple hemogram tests in patients with UGIB, in terms of clinical use and as a mortality marker.

Materials and methods: The patients diagnosed with UGIB between March 1, 2019, and September 30, 2019, were evaluated retrospectively. Demographic characteristics, causes of hemorrhage, clinical presentations, hemogram, and biochemistry values at ED admission and 30-day mortality status of the patients were examined. We divided the patients into groups according to their mortality status, and the groups were compared among themselves in terms of parameters.

Results: A total of 213 patients who met the inclusion criteria were included in the study. Of these patients, 139 (65.3\%) were male and the mean age was $65.05 \pm 16.7$ years. Fifteen (7\%) of them were in the nonsurvival group, while $198(93 \%)$ were in the survival group. The efficacy of both the IG count (IGC) and IG\% in predicting mortality was statistically significant ( $p=0.002, p=0.008$, respectively). The sensitivity and specificity for the IGC were found as $60 \%$ and 84.4 ; for the IG\%, they were found as $66.7 \%$ and $75.7 \%$, respectively.

Conclusion: IGC and IG\% are independent risk factors for the 30-day mortality status. These measurements are obtained from simple hemogram tests and may be useful for the evaluation of mortality in patients with UGIB.

Keywords: Hemorrhage, Immature granulocyte, Upper gastrointestinal bleeding.

Indian Journal of Critical Care Medicine (2020): 10.5005/jp-journals-10071-23563
\end{abstract}

\section{INTRODUCTION}

Upper gastrointestinal bleeding (UGIB) is bleeding into the lumen from anywhere between the upper part of the esophagus and the Treitz ligament, and is one of the important causes of admissions to the ED. ${ }^{1}$ Despite improvements in diagnosis and treatment methods, its annual incidence is $50-180$ per 100,000 population, the overall mortality rate is around $10 \%$, and this rate is estimated to reach $35 \%$ in the patients with comorbidities. ${ }^{1,2}$ The early diagnosis of the patients at risk for the poor outcomes can be difficult to predict in emergency situations. ${ }^{3,4}$

Multiple clinical factors, including advanced age, lifestyle, melena, comorbid diseases, and laboratory markers such as uremia and low hemoglobin $(\mathrm{Hg})$, are known to be associated with a high risk of morbidity and mortality. ${ }^{1}$ Various scoring systems have been developed in recent years for the risk assessment of the patients with UGIB, and the most common ones are the Glasgow blatchford scoring (GBS) and the Rockall Scoring systems. The GBS uses basic clinical and laboratory variables in its application, while the Rockall score is a score with endoscopic components as well. ${ }^{5,6}$ Although these scoring systems have a place in clinical practice, it can be difficult for physicians to assess the detection and hospitalization of critical patients quickly, easily, and accurately in the ED. Continuous monitoring of vital signs and laboratory tests are often required. ${ }^{7,8}$

Massive bleeding and hemorrhagic damage lead to inflammation, and a sustained, flaring inflammatory response is strongly associated with increased mortality. ${ }^{1-3}$ Immature granulocyte (IG) reflects the fraction of IGs in the peripheral blood; it is an indicator of the bone marrow activation and serious infection, but it is not a parameter that is sufficiently known by

\begin{abstract}
1,2Department of Emergency Medicine, Health Science University Antalya Training and Research Hospital, Antalya, Turkey

${ }^{3}$ Department of Emergency Medicine, Karaman State Hospital, Karaman, Turkey

${ }^{4}$ Department of Emergency Medicine, Karabük University Training and Research Hospital, Karabük, Turkey

Corresponding Author: Cihan Bedel, Department of Emergency Medicine, Health Science University Antalya Training and Research Hospital, Antalya, Turkey, Phone:+905075641254,e-mail:cihanbedel@ hotmail.com

How to cite this article: Bedel C, Korkut M, Avcl A, Uzun A. Immature Granulocyte Count and Percentage as New Predictors of Mortality in Patients with Upper Gastrointestinal Bleeding. Indian J Crit Care Med 2020;24(9):794-798.
\end{abstract}

Source of support: Nil

Conflict of interest: None

many clinicians. ${ }^{9,10}$ With the latest technological developments, IG can be measured easily and quickly on automated blood cell analyzers. ${ }^{11}$ Studies have shown that IG is useful for predicting the severity of many disease processes, including gastrointestinal tract diseases. In addition, increased IG has been reported to be strongly associated with systemic inflammation and mortality. ${ }^{11-13}$ The role of IG in UGIB patients in terms of clinical utility and as a marker for mortality has not yet been investigated. Therefore, the aim of the study is to evaluate IG, which can be obtained from simple hemogram tests in patients with UGIB, in terms of clinical use and as a mortality marker. 


\section{Materials and Methods}

The patients admitted to the tertiary ED and diagnosed with UGIB between March 1, 2019, and September 30, 2019, were retrospectively screened through the hospital informationrecording system. Upper gastrointestinal bleeding was defined as the hemorrhage into the lumen between the proximal duodenum up to the Treitz ligament and the upper esophageal sphincter. ${ }^{1}$ Patients who were admitted to the ED with at least one of the symptoms of melena, hematochezia, hematemesis, vomiting in the form of coffee grounds, and blood in the nasogastric aspirate were defined as the patients with UGIB. ${ }^{1-3}$

This study included 284 patients suffering from UGIB, who were applied to our ED. Patients who were under 18 years of age, pregnant, with hematological malignancies that could change hematological parameters, using granulocyte colony-stimulating factor, immunosuppressive agent or steroid, and receiving blood transfusions before admission, who transferred to other hospitals, who had lower gastrointestinal bleeding, underlying infectious disease and trauma were receiving chemotherapy within 2 weeks, and whose data were incomplete were excluded from the study. A total of 71 patients were excluded. Consequently, 213 patients complying with these criteria were selected for this study.

Demographic characteristics, causes of hemorrhage, clinical presentations at ED admission, vital signs, comorbid diseases, drug use, habits, hemogram and biochemistry values at ED admission, results of endoscopy if performed during hospitalization, length of hospital stay (LOS), and 30-day mortality status of the patients were examined. In accordance with the treatment protocols of our hospital, all the blood is collected within 30 minutes at the time of ED application. Of the laboratory parameters of the patients, the immature granulocyte count (IGC), IG percentage (IG\%), white blood cell, neutrophil, lymphocyte, platelet, hemoglobin, hematocrit, and C-reactive protein (CRP) data were used, and of the biochemistry parameters, blood urea nitrogen (BUN), creatinine, and urea were used. The INR values were also recorded. The Rockall score was calculated based on age, shock status, comorbidity, endoscopy finding, and major bleeding status. ${ }^{5}$ The GBS was calculated based on the clinical and laboratory findings of the disease (urea, hemoglobin, comorbid diseases, blood pressure, and type of admission). ${ }^{6}$ We divided the patients into groups according to their mortality status, and the groups were compared.

\section{Data Analysis}

Standard deviation and mean values were calculated for continuous variables, and median and interquartile range were calculated for nonparametric data. The descriptive statistical analysis of all variables was carried out using SPSS 18.0. Logistic regression was conducted for factors associated with mortality. The optimum cut-off value of the IGC and IG\% examined in the ED for mortality in the patients with UGIB was analyzed by the receiver operating characteristic $(\mathrm{ROC})$ analysis.

\section{Results}

A total of 213 patients who met the inclusion criteria were included in the study. Of these patients, 139 (65.3\%) were male, and the mean age was $65.05 \pm 16.7$ years. The patients were divided into two groups as the nonsurvival and survival groups according to the 30 -day mortality status. Fifteen (7\%) of them were in the nonsurvival group, while 198 (93\%) were in the survival group.
In terms of gender, there was no significant difference between the groups ( $p=0.779$ ). The mean age was statistically significantly higher in patients with mortality than those without mortality $(p=0.004)$. The calculated Rockall and Glasgow-Blatchford scores of the patients with 30-day mortality were statistically significantly higher ( $p=0.00, p=0.038$, respectively). According to the vital signs of the patients, systolic and diastolic blood pressures were lower and the heart rate was higher in the nonsurvival group compared to the survival group. Of the patients, 146 (68.5\%) were admitted to the EDwith hematemesis, 27 (12.6\%) with melena, and 40 (18.9\%) with hematochezia.

When the groups were compared according to comorbidity status, the percentage of mortality was significantly higher in patients with malignancy ( $p=0.012$ ). The mean white blood cell (WBC), neutrophil, creatinine, CRP, IGC, and IG\% levels were significantly higher in the nonsurvival group compared to the survival group ( $p<0.05$ for all markers). When the two groups were compared in terms of LOS, the mean LOS was 4 days in the survival group, while it was 7 days in the nonsurvival group, and this finding was statistically significant $(p=0.01)$. Demographic data, etiologic characteristics, and laboratory values of the groups were compared in Table 1.

In the multivariate analysis, age, neutrophil, BUN IG\% and IGC are the independent predictors of the 30-day mortality in patients with UGIB (Table 2). The efficacy of the IGC and IG\% parameters in determining mortality was calculated by plotting ROC curves (Fig. 1). The efficacy of both the IGC and IG\% in predicting mortality was statistically significant ( $p=0.002, p=0.008$, respectively). The AUC, sensitivity, and specificity for the IGC were found as 0.735 , $60 \%$, and $84.4 \%$ at the cut-off value of 0.17 , respectively; for the IG\%, they were found as $0.705,66.7 \%$, and $75.7 \%$ at the cut-off value of 0.95 , respectively. The ROC analysis results are presented in Table 3.

\section{Discussion}

Upper gastrointestinal bleeding is among the common causes of mortality and morbidity. Early diagnosis and correct classification of the patients with a high risk of mortality and rebleeding increase the care efficiency of the patients and may help their possible outcome. ${ }^{14}$ Mortality has been shown to be associated with many risk factors in the patients with UGIB, such as advanced age, malignancy, and abnormal vital signs. ${ }^{15}$ The pathophysiological mechanism of UGIB, leading to mortality, is not clearly understood. ${ }^{14}$ Studies have shown that the amount of bleeding or shock in UGIB is associated with the production of many proinflammatory factors including interleukin-6, interleukin-8, and CRP. Many researchers have proposed mechanisms to explain this rapid and early release of IG, a large amount of blood is lost from the body, including neutrophils..$^{12-17}$ In addition, it has been recognized in recent years that the widespread inflammation may also lead to downregulation of immune responses. As a result, the host is highly susceptible to infections. In addition, dysregulation of immune mechanisms can increase the death rate. ${ }^{18}$ We showed in our study that the 30-day mortality can be predicted with high IGC and IG\% levels in the patients with UGIB.

Previous studies have proposed several mechanisms to explain this rapid release of IG, and the hematopoietic system can quickly adapt to hematopoietic stress from steady state to severe granulopoiesis, such as severe infection or bleeding..$^{12}$ IGC and IG\% may reflect the severity of systemic inflammation. Therefore, our findings suggest that the IGC and IG\% can predict mortality 
New Predictors of Mortality in Patients with UGIB

Table 1: Baseline characteristics of patients stratified according to the 30-day mortality

\begin{tabular}{|c|c|c|c|c|}
\hline Variables & Total $(n=213)$ & Death $(n=15)$ & Survival $(n=198)$ & $p$ \\
\hline Age (years) & $65.05 \pm 16.7$ & $76.33 \pm 11.33$ & $64.38 \pm 16.68$ & 0.004 \\
\hline Male gender $n(\%)$ & $139(65.3)$ & $9(60)$ & $130(65.3)$ & 0.779 \\
\hline Rockall score & $2.66 \pm 1.89$ & $4.93 \pm 1.98$ & $2.49 \pm 1.77$ & 0.000 \\
\hline Glasgow-Blatchford score & $9.74 \pm 3.97$ & $11.6 \pm 3.39$ & $9.6 \pm 3.97$ & 0.038 \\
\hline \multicolumn{5}{|l|}{ Vital signs at ED presentation } \\
\hline Systolic blood pressure $(\mathrm{mm} \mathrm{Hg})$ & $118(70-180)$ & $100(80-180)$ & $120(70-146)$ & 0.006 \\
\hline Diastolic blood pressure $(\mathrm{mm} \mathrm{Hg})$ & $70(40-118)$ & $62(40-90)$ & $72(40-118)$ & 0.002 \\
\hline Heart rate (bpm) & $88(58-148)$ & $106(76-140)$ & $88(58-148)$ & 0.002 \\
\hline Presenting symptoms & & & & 0.159 \\
\hline Hematemesis & $146(68.5)$ & $7(46.7)$ & $139(70.2)$ & \\
\hline Melena & $27(12.6)$ & $2(13.3)$ & $25(12.6)$ & \\
\hline Hematochezia & $40(18,9)$ & $6(40)$ & $34(17.2)$ & \\
\hline \multicolumn{5}{|l|}{ Comorbidity } \\
\hline Hypertension & $87(40.8)$ & $9(60)$ & $78(39.6)$ & 0.172 \\
\hline Diabetes mellitus & $47(22.1)$ & $5(33.3)$ & $42(21.2)$ & 0.330 \\
\hline Liver cirrhosis & $9(4.2)$ & $1(6.7)$ & $8(4)$ & 0.488 \\
\hline Cerebrovascular disease & $18(8.7)$ & $2(13.3)$ & $16(8.3)$ & 0.625 \\
\hline Malignancy & $27(12.7)$ & $6(40)$ & $21(10.6)$ & 0.012 \\
\hline Previous UGI bleeding & $10(4.6)$ & $2(13.3)$ & $8(4)$ & 0.248 \\
\hline Forrest classification & & & & 0.987 \\
\hline IB (Oozing) & $67(31.4)$ & $5(33.3)$ & $62(31.3)$ & \\
\hline IA (active hemorrhage) & $46(22.1)$ & $4(26.7)$ & $43(21.7)$ & \\
\hline II-III & $71(33.3)$ & $6(40)$ & $65(32.8)$ & \\
\hline \multicolumn{5}{|l|}{ Laboratory features } \\
\hline White blood cell $\left(10^{3} / \mathrm{mL}\right)$ & $11.25 \pm 7.62$ & $14.96 \pm 6.62$ & $11 \pm 7.63$ & 0.006 \\
\hline Neutrophil $\left(10^{3} / \mathrm{mL}\right)$ & $7.99 \pm 6.14$ & $12.04 \pm 6.45$ & $7.72 \pm 6.03$ & 0.004 \\
\hline Lymphocyte $\left(10^{3} / \mathrm{mL}\right)$ & $2.36 \pm 2.73$ & $2.904 \pm 1.44$ & $2.39 \pm 1.81$ & 0.46 \\
\hline Hemoglobin (g/L) & $9.44 \pm 3.01$ & $9.25 \pm 2.96$ & $9.44 \pm 3.33$ & 0.931 \\
\hline Hematocrit (\%) & $28,28 \pm 7,72$ & $27.4 \pm 8.51$ & $28.28 \pm 8.78$ & 0.791 \\
\hline Platelet (103/mL) & $253.27 \pm 111.95$ & $244.73 \pm 129.77$ & $253.85 \pm 111.12$ & 0.764 \\
\hline BUN (mg/dL) & $35.66 \pm 22.35$ & $55.21 \pm 28.62$ & $34.4 \pm 21.4$ & 0.001 \\
\hline Creatinine (mg/dL) & $1.3 \pm 1.08$ & $1.72 \pm 1.19$ & $1.28 \pm 1.01$ & 0.06 \\
\hline CRP & $24.19 \pm 40.22$ & $56.92 \pm 42.1$ & $21.99 \pm 15.25$ & 0.001 \\
\hline IG (\%) & $1.23 \pm 1.98$ & $2.12 \pm 2.24$ & $1.17 \pm 1.95$ & 0.008 \\
\hline IGC & $0.16 \pm 0.36$ & $0.5 \pm 0.69$ & $0.14 \pm 0.31$ & 0.002 \\
\hline Length of stay (day) & $5(1-39)$ & $7(1-28)$ & $4(1-39)$ & 0.01 \\
\hline
\end{tabular}

IGC, immature granulocyte count; IG\%, immature granulocyte percentage; CRP, C-reactive protein; BUN, blood urea nitrogen

Table 2: Logistic regression analysis of risk factors affected UGIB 30-day mortality

\begin{tabular}{llllll}
\hline & \multicolumn{2}{c}{ Univariate analysis } & & \multicolumn{2}{c}{ Multivariate analysis } \\
\cline { 2 - 3 } \cline { 5 - 6 } Variables & $O R(95 \% \mathrm{Cl})$ & $p$ value & & $O R(95 \% \mathrm{Cl})$ & $\mathrm{O}$ value \\
\hline Age & $2.118(1.981-$ & $<0.001$ & & $2.863(1.023-$ & $<0.001$ \\
& $2.256)$ & & & $3.012)$ & \\
CRP & $0.02(0.15-0.25)$ & $<0.001$ & & $0.401(0.21-0.65)$ & 0.041 \\
IG (\%) & $0.320(0.180-$ & $<0.001$ & & $0.371(0.081-$ & $<0.001$ \\
& $0.461)$ & & & $1.698)$ & \\
IGC & $1.888(1.277-$ & $<0.001$ & & $1.240(0.661-$ & $<0.001$ \\
& $2.499)$ & & $3.545)$ & \\
\hline
\end{tabular}

IGC, immature granulocyte count; IG\%, immature granulocyte percentage; CRP, C-reactive protein in patients with UGIH. Studies have indicated that the IGC and IG\% reflect an infection-related response and may be associated with mortality. ${ }^{11}$ Studies on the role of inflammatory biomarkers in the assessment of massive bleeding have increased in recent years. In a present study, a positive correlation between increased CRP values and gastrointestinal bleeding has been shown. ${ }^{19}$ In a recent study, they found that the neutrophil lymphocyte ratio (NLR) was significantly higher in patients with gastrointestinal bleeding compared to the control group. ${ }^{20}$ The platelet lymphocyte ratio (PLR) was also significantly higher in those with Gl bleeding compared to those without GI bleeding. IG and its derivatives are known to be new systemic inflammation biomarkers expressed in various conditions such as systemic inflammation. ${ }^{11,21}$ A study 


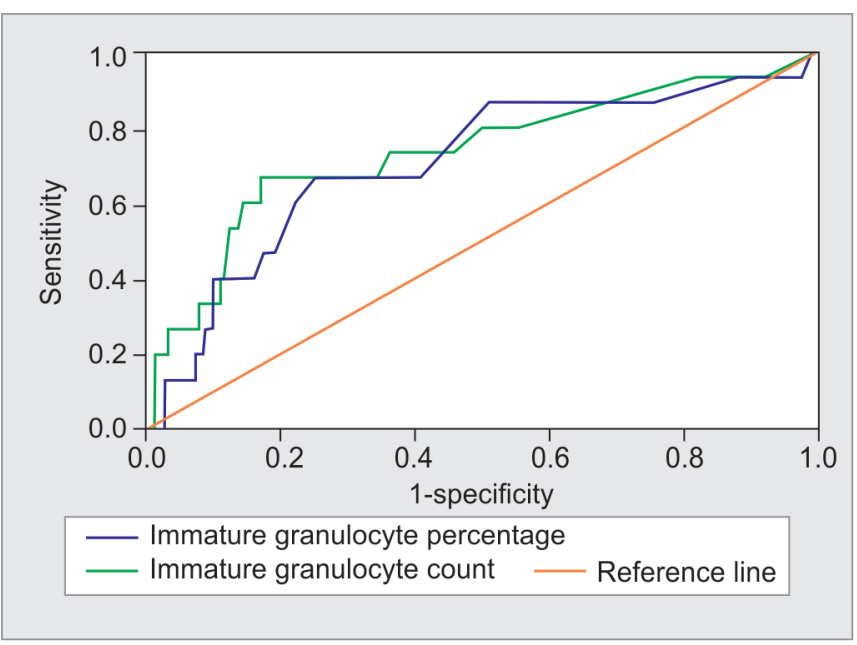

Fig. 1: Receiver operating characteristic curve analysis of IGC and IG\% parameters in the discrimination between mortality

Table 3: Diagnostic accuracy of prognostic parameters to predict UGIB 30-day mortality with the best predictive cut-off values

\begin{tabular}{lllllll}
\hline & AUC & $\begin{array}{l}\text { Cut-off } \\
\text { value }\end{array}$ & $\begin{array}{l}\text { Sensitivity } \\
(\%)\end{array}$ & $\begin{array}{l}\text { Specificity } \\
\text { (\%) }\end{array}$ & $95 \% \mathrm{Cl}$ & $\begin{array}{l}p \\
\text { value }\end{array}$ \\
\hline IG\% & 0.705 & 0.95 & 66.7 & 75.7 & $0.559-0.851$ & 0.008 \\
IGC & 0.735 & 0.17 & 60 & 84.4 & $0.585-0.884$ & 0.002 \\
\hline
\end{tabular}

AUC, area under the curve; IG\%, immature granulocyte percentage; IGC, immature granulocyte count

states that the IG\% showed appendicitis complications with low sensitivity and high specificity. ${ }^{22} \mathrm{~A}$ study by Kong et al. ${ }^{23}$ conducted with trauma patients showed that IG\% could be a marker of the 30 -day mortality at $>5.3 \%$ value. In another study, they found that severe sepsis and septic shock significantly increased in patients with an IG value $>6.5 \% .{ }^{24} \mathrm{~A}$ recent study has reported a positive correlation between increasing IG values and cardiac arrest. ${ }^{25}$ The increase in the number of IGs in circulation thought to endanger innate immunity. In our study, IGC and IG\% were important independent risk factors for mortality in UGIB patients. The IGC and IG\%, which are quick, easy, and affordable measurement methods, can be used to assess the severity of various diseases as a part of complete blood count. Similarly, we think that IGC and IG\% have similar clinical results in UGIB patients, considering our study results which support their use in the risk classification of these patients.

Similar to the literature, known risk factors for mortality, such as malignancy and the Rockall score, were found to have an effect on mortality for UGIB in this study. ${ }^{26}$ In addition, the mean WBC, neutrophil, creatinine, CRP, IGC, and IG\% levels were associated with mortality. The IGC and IG\% are the predictors of the 30-day mortality in patients with UGIB, and these parameters can simply be obtained from hemogram tests and used for the risk assessment.

In previous studies, factors such as hemodynamic instability, comorbid diseases, a hemoglobin value less than $10 \mathrm{~g} / \mathrm{L}$, active bleeding in endoscopy were used as parameters for the risk assessment. ${ }^{27}$ Barkun et al. ${ }^{28}$ reported in their study that an SBP value $<100 \mathrm{~mm} \mathrm{Hg}$, a hemoglobin value less than $10 \mathrm{~g} / \mathrm{L}$, and blood transfusion were the risk factors for excessive bleeding before endoscopy. The Rockall score was found to be associated with more bleeding in our study. Therapeutic decisions such as emergency endoscopic evaluation or blood transfusion of the patients with UGIB vary depending on the prognosis and the severity of the disease. Risk scoring systems such as GBS and Rockall score are used to predict disease mortality. ${ }^{5,29}$ Although recent studies have revealed that the NLR and PLR are associated with the severity of UGIB, there are very few studies in the literature suggesting IGC and IG\%. ${ }^{30,31}$ Our study showed that the IGC and IG\%, new independent parameters, have been shown to predict mortality and may play a role in improving the risk classification of UGIB patients.

Rockall and Glasgow Blatchford scores, significantly higher mean WBC, neutrophil, creatinine, CRP, IGC, and IG\% levels in the nonsurvival group, significant differences in the LOS, findings of multivariate logistic regression analysis, efficacy of both the IGC and IG\% in predicting mortality, etc. are the major findings of the current study.

\section{Conclusion}

The IGC and IG\% are independent risk factors for the 30-day mortality status, and these measurements are obtained from the simple hemogram tests and may be useful for the the evaluation of mortality in patients with UGIB. The IGC and IG\% can be included as parameters for further development of risk scoring systems.

\section{Limitation}

There are some limitations of this study. First, some treatment options might differ among the cases where the study was conducted. Second, our study was conducted in a single institution with a relatively small number of participants. Third, there was a risk of selection bias in this study, as the patients did not have a serial IGC and IG\% assessment. In addition, our study had another important limitation that the diagnosis and treatment were not determined by the same physicians.

\section{Ethics Approval}

The study was approved by the institutional ethics committee.

\section{ACKNOWLedgments}

We gratefully acknowledge the help and support of all staff of the Department of Emergency Medicine, Antalya Training, and Research Hospital.

\section{References}

1. Hajiagha Mohammadi AA, Reza Azizi M. Prognostic factors in patients with active non-variceal upper gastrointestinal bleeding. Arab J Gastroenterol 2019;20(1):23-27. DOI: 10.1016/j.ajg.2019.01.001.

2. Fouad TR, Abdelsameea E, Abdel-Razek W, Attia A, Mohamed A, Metwally K, et al. Upper gastrointestinal bleeding in Egyptian patients with cirrhosis: post-therapeutic outcome and prognostic indicators.J Gastroenterol Hepatol 2019;34(9):1604-1610. DOI: 10.1111/jgh.14659.

3. Shah A, Chisolm-Straker M, Alexander A, Rattu M, Dikdan S, Manini AF. Prognostic use of lactate to predict inpatient mortality in acute gastrointestinal hemorrhage. Am J Emerg Med 2014;32(7):752-755. DOI: 10.1016/j.ajem.2014.02.010.

4. Badipatla KR, Jadhav P, Vaddigiri S, Bajantri B, Singh A, Chandrala $C$, et al. Predictors of acute gastrointestinal bleeding in diabetic ketoacidosis: a retrospective observational study in minority population. Gastroenterol Rep 2017;5(4):293-297. DOI: 10.1093/ gastro/gox006.

5. Monteiro S, Gonçalves TC, Magalhães J, Cotter J. Upper gastrointestinal bleeding risk scores: who, when and why? World J Gastrointest Pathophysiol 2016;7(1):86-96. DOI: 10.4291/wjgp.v7.i1.86. 
6. Shahrami A, Ahmadi S, Safari S. Full and modified Glasgow-Blatchford bleeding score in predicting the outcome of patients with acute upper gastrointestinal bleeding; a diagnostic accuracy study. Emergency 2018;6(1):e31.

7. Ko B, Kim YJ, Jung D, Sohn C, Seo D, Lee YS, et al. Early risk score for predicting hypotension in normotensive patients with non-variceal upper gastrointestinal bleeding. J Clin Med 2019;8(1):37. DOI: 10.3390/ jcm8010037.

8. Sauneuf B, Bouffard C, Cornet E, Daubin C, Desmeulles I, Masson $\mathrm{R}$, et al. Immature/total granulocyte ratio: a promising tool to assess the severity and the outcome of post-cardiac arrest syndrome. Resuscitation 2014;85(8):1115-1119. DOI: 10.1016/j. resuscitation.2014.04.017.

9. Koseoglu Z, Ozkan O, Semerci E, Aslan A, Yetim I, Ucar E, et al. The relationship between mortality and inflammation in patients with gastrointestinal bleeding. J Int Med Res 2009;37(5):1508-1514. DOI: 10.1177/147323000903700528.

10. Lu S, Aguilar A, Subramani K, Poulose N, Ayub A, Raju R. Alteration of cytokine profile following hemorrhagic shock. Cytokine 2016;81:3538. DOI: 10.1016/j.cyto.2016.01.022.

11. Karon BS, Tolan NV, Wockenfus AM, Block DR, Baumann NA, Bryant SC, et al. Evaluation of lactate, white blood cell count, neutrophil count, procalcitonin and immature granulocyte count as biomarkers for sepsis in emergency department patients. Clin Biochem 2017;50(1617):956-958. DOI: 10.1016/j.clinbiochem.2017.05.014.

12. Huang $Y$, Xiao J, Cai T, Yang L, Shi F, Wang Y, et al. Immature granulocytes: a novel biomarker of acute respiratory distress syndrome in patients with acute pancreatitis. J Crit Care 2019;50:303308. DOI: $10.1016 /$ j.jcrc.2018.12.002.

13. Ayres LS, Sgnaolin V, Munhoz TP. Immature granulocytes index as early marker of sepsis. Int J Lab Hematol 2019;41(3):392-396. DOI: 10.1111/ijlh.12990.

14. Kolaczkowska E, Kubes P. Neutrophil recruitment and function in health and inflammation. Nat Rev Immunol 2013;13(3):159-175. DOI: 10.1038/nri3399.

15. Stanley AJ, Laine L, Dalton HR, Ngu JH, Schultz M, Abazi R, et al. Comparison of risk scoring systems for patients presenting with upper gastrointestinal bleeding: international multicentre prospective study. BMJ 2017;356:i6432. DOI: 10.1136/bmj.i6432.

16. Tsai JP, Lee CJ, Subeq YM, Lee RP, Hsu BG. Calcitriol decreases proinflammatory cytokines and protects against severe hemorrhagic shock induced-organ damage in rats. Cytokine 2016;83:262-268. DOI: 10.1016/j.cyto.2016.05.008.

17. Mortaz E, Alipoor SD, Adcock IM, Mumby S, Koenderman L. Update on neutrophil function in severe inflammation. Front Immunol 2018;9:2171. DOI: 10.3389/fimmu.2018.02171.

18. Sônego F, Castanheira FVS, Ferreira RG, Kanashiro A, Leite CAVG, Nascimento DC, et al. Paradoxical roles of the neutrophil in sepsis: protective and deleterious. Front Immunol 2016;7:155. DOI: 10.3389/ fimmu.2016.00155.

19. Tomizawa M, Shinozaki F, Hasegawa R, Shirai $Y$, Motoyoshi $Y$, Sugiyama $\mathrm{T}$, et al. Elevated C-reactive protein level predicts lower gastrointestinal tract bleeding. Biomed Rep 2016;4(6):711-714. DOI 10.3892/br.2016.654.

20. Gayret OB, Erol M, Tekin Nacaroglu H. The relationship of neutrophillymphocyte ratio and platelet-lymphocyte ratio with gastrointestinal bleeding in henoch-schonlein purpura. Iran J Pediatr 2016;26(5):8191. DOI: $10.5812 /$ ijp. 8191 .

21. Nahm CH, Choi JW, Lee J. Delta neutrophil index in automated immature granulocyte counts for assessing disease severity of patients with sepsis. Ann Clin Lab Sci 2008;38(3):241-246.

22. Rhee NG, Kim KM, Kim HJ, Chung SP, Lee HS, Lee JW. Assessment of perforation of acute appendicitis using the delta neutrophil index reflecting peripheral immature granulocyte count. J Korean Soc Emerg Med 2012;23:389-393.

23. Kong T, Park YS, Lee HS, Kim S, Lee JW, You JS, et al. The delta neutrophil index predicts development of multiple organ dysfunction syndrome and 30-day mortality in trauma patients admitted to an intensive care unit: a retrospective analysis. Sci Rep 2018;8(1):17515. DOI: 10.1038/ s41598-018-35796-4.

24. Park BH, Kang YA, Park MS, Jung WJ, Lee SH, Lee SK, et al. Delta neutrophil index as an early marker of disease severity in critically ill patients with sepsis. BMC Infect Dis 2011;11(1):299. DOI: 10.1186/14712334-11-299.

25. Yune HY, Chung SP, Park YS, Chung HS, Lee HS, Lee JW, et al. Delta neutrophil index as a promising prognostic marker in out of hospital cardiac arrest. PLoS ONE 2015;10(3):120677. DOI: 10.1371/journal. pone.0120677.

26. Martinez-Cara JG, Jimenez-Rosales R, Ubeda-Munoz M, de Hierro ML, de Teresa J, Redondo-Cerezo E. Comparison of AIMS65, GlasgowBlatchford score, and Rockall score in a European series of patients with upper gastrointestinal bleeding: performance when predicting in-hospital and delayed mortality. United Eur Gastroenterol J 2016;4(3):371-379. DOI: 10.1177/2050640615604779.

27. García-Iglesias P, Villoria A, Suarez D, Brullet E, Gallach M, Feu F, et al. Meta-analysis: predictors of rebleeding after endoscopic treatment for bleeding peptic ulcer. Aliment Pharmacol Ther 2011;34(8):888900. DOI: 10.1111/j.1365-2036.2011.04830.x.

28. Barkun AN, Bardou M, Martel M, Gralnek IM, Sung JJ. Prokinetics in acute upper GI bleeding: a meta-analysis. Gastrointest Endosc 2010;72(6):1138-1145. DOI: 10.1016/j.gie.2010.08.011.

29. Cheng DW, Lu YW, Teller T, Sekhon HK, Wu BU. A modified Glasgow blatchford score improves risk stratification in upper gastrointestinal bleed: a prospective comparison of scoring systems. Aliment Pharmacol Ther 2012;36(8):782-789. DOI: 10.1111/apt.12029.

30. Kong T, In S, Park YS, Lee HS, Lee JW, You JS, et al. Usefulness of the delta neutrophil index to predict 30-day mortality in patients with upper gastrointestinal bleeding. Shock 2017;48(4):427-435. DOI: 10.1097/SHK.0000000000000878.

31. Zou Y, Zhang W, Huang C, Zhu Y. Clinical significance of neutrophil to lymphocyte ratio and platelet to lymphocyte ratio in acute cerebral hemorrhage with gastrointestinal hemorrhage, and logistic regression analysis of risk factors. Exp Ther Med 2019;18(3):1533-1538. DOI: 10.3892/etm.2019.7778. 